Published in final edited form as:

Trends Mol Med. 2011 February ; 17(2): 57-64. doi:10.1016/j.molmed.2010.11.001.

\title{
Inflammasomes and autoimmunity
}

\author{
Patrick J. Shaw ${ }^{1}$, Michael F. McDermott ${ }^{2}$, and Thirumala-Devi Kanneganti ${ }^{1}$ \\ ${ }^{1}$ Department of Immunology, St. Jude Children's Research Hospital, MS \#351, Suite E7004, 262 \\ Danny Thomas Place, Memphis, Tennessee 38105, USA \\ ${ }^{2} \mathrm{NIHR}$-Leeds Musculoskeletal Biomedical Research Unit (NIHR-LMBRU), Leeds Institute of \\ Molecular Medicine, Wellcome Trust Brenner Building, St. James's University Hospital, Leeds, \\ LS9 7TF, UK
}

\begin{abstract}
The NOD-like receptor (NLR) family members are cytosolic sensors of microbial components and danger signals. A subset of NLRs control inflammasome assembly that results in caspase-1 activation and, in turn, IL-1 $\beta$ and IL-18 production. Excessive inflammasome activation can cause autoinflammatory disorders, including the hereditary periodic fevers. Autoinflammatory and autoimmune diseases form a disease spectrum of aberrant, immune-mediated inflammation against self, through innate and adaptive immunity. However, the role of inflammasomes in autoimmune disease is less clear than in autoinflammation, despite the numerous effects IL- $1 \beta$ and IL-18 can have on shaping adaptive immunity. We summarize the role of inflammasomes in autoimmune disorders, highlight the need for a better understanding of inflammasomes in these conditions and offer suggestions for future research directions.
\end{abstract}

\section{Keywords}

NLR; Inflammasome; NLRP3; ASC; caspase-1; autoimmunity

\section{NLR-induced assembly of the inflammasome}

Multicellular organisms have developed an intricate network of innate and adaptive signals to mount effective responses to both endogenous and exogenous insults. The Nod-like receptor (NLR) family of proteins is a group of intracellular pattern recognition receptors (PRRs) of the innate immune system that play a vital role in the recognition of a wide spectrum of danger- and pathogen-associated molecular patterns (DAMPs and PAMPs, respectively) 1. In humans, the NLR family is composed of 22 genes, whereas the mouse genome contains at least 34 NLR-encoding genes2. Of the NLR proteins, NLRP3 (also known as NALP3 or cryopyrin), NLRP1, NLRC4 and absence in melanoma 2 (AIM2)

\footnotetext{
(C) 2010 Elsevier Ltd. All rights reserved.

Address correspondence to: Thirumala-Devi Kanneganti, Department of Immunology, MS 351, St. Jude Children's Research Hospital, Memphis, TN 38104, Tel: (901) 595-3634, Fax: (901) 595-5766, thirumala-devi.kanneganti@stjude.org.

Publisher's Disclaimer: This is a PDF file of an unedited manuscript that has been accepted for publication. As a service to our customers we are providing this early version of the manuscript. The manuscript will undergo copyediting, typesetting, and review of the resulting proof before it is published in its final citable form. Please note that during the production process errors may be discovered which could affect the content, and all legal disclaimers that apply to the journal pertain.

Competing interest statement

The author declares no competing financial interests.

Further Information

Thirumala-DeviKanneganti's home page: http://www.stjude.org/Kanneganti
} 
activation results in the formation of large protein complexes termed inflammasomes. The mechanism of activation of NLRs is still a subject of active debate, but once activated NLRP3, NLRP1, NLRC4 and AIM2 undergo a conformational change allowing for interaction with an inflammasome-adaptor protein, ASC (PYCARD), which, in turn, interacts with caspase-1 (NLRP3 is shown in Figure 1 as an example). The resulting inflammasome serves as a molecular platform that mediates the autoactivation of caspase-1, which cleaves the pro-forms of IL- $1 \beta$ and IL-18 to active forms. Inflammasome activation is critical for host defense to pathogens, but recent work has also found a role for the inflammasome in the pathogenesis of several diseases with an inflammatory component, such as type 2 diabetes (T2D), inflammatory bowel disease (IBD) and atherosclerosis ${ }^{3-} 6$ (Table 1). However, this review will focus on the role of inflammasomes in diseases characterized by inflammation against self, highlighting autoimmune diseases in particular.

\section{Inflammasome activation and the continuum of diseases caused by immune-mediated inflammation against self}

Diseases classified by excessive or chronic activation of the immune system can be placed in a disease continuum, with autoinflammatory disorders on one end of the spectrum and autoimmune diseases at the other7. Autoinflammatory diseases are clinical disorders that present with recurrent inflammation and unexplained fevers as part of their phenotype, due to abnormally increased inflammation mediated by cells of the innate immune system ${ }^{8}$. In autoinflammatory disorders, tissue damage results from self-directed inflammation, due to activation of innate immune cells, including macrophages and neutrophils. For example, disturbed homeostasis of cytokine cascades in the periodic fevers, and aberrant bacterial sensing, as in Crohn's disease, predispose to site-specific inflammation that is largely independent of adaptive immune responses. By contrast, autoimmune diseases can be classified as inflammation against self mediated by the adaptive immune system, with development of immune reactivity towards native antigens. Hyper-reactivity of both $\mathrm{T}$ and $\mathrm{B}$ cells (as well as aberrant dendritic cells) is typically observed in conjunction with autoantibodies and antigen-specific T cells targeting self, resulting in tissue destruction. Autoimmune diseases can cause multiorgan involvement, but the primary end-organ target typically drives the clinical presentation and disease definition.

To date, several prototypical autoinflammatory disorders have been strongly linked with mutations in inflammasome-forming NLRs9. Overactivating mutations in the NLRP3 inflammasome increase the release of IL-1 $\beta$ and cause cryopyrin-associated periodic syndromes (CAPS) ${ }^{10}$. Familial cold autoinflammatory syndrome (FCAS), Muckle-Wells syndrome (MWS) and Neonatal Onset Multisystem Inflammatory Disease (NOMID) syndrome comprise the various CAPS caused by NLRP3 gene mutations, of which over 50 different mutations have been identified 9 , 10 . The three CAPS conditions have clinical similarities but are distinguished by their phenotypic severity. Signs and symptoms of these disorders include recurrent rash, fever/chills, joint pain, fatigue, deafness, systemic amyloidosis, CNS disabilities, vision loss and joint and bone deformation. Direct, continuous inhibition of IL-1 $\beta$ by anakinra, rilonacept or canakinumab ameliorates all clinical manifestations, except bony overgrowth in NOMID syndrome11, 12 .

The NLRP3 inflammasome has also been linked with polygenic inflammatory disorders, such as gout and pseudogout, which are dependent on the synergistic interaction between free fatty acids and monosodium urate crystals driving inflammation through the NLRP3 inflammasome ${ }^{13}$. The resulting inflammation leads to neutrophil and macrophage activation, especially in the joints ${ }^{14}$. Blockade of IL-1 $\beta$ with anakinra has a dramatic effect, exemplifying the critical role of inflammasome activation in disease progression ${ }^{15}$. 
The understanding of the role of inflammasomes in autoimmunity is less clear. The innate immune system influences the development of the autoimmune response ${ }^{16}$, and a T cellindependent phase has been postulated for the initiation of rheumatoid arthritis (RA), a systemic autoimmune disease ${ }^{17}$. Genetic links between the inflammasome-inducing NLRs and autoimmune diseases are limited, but this does not exclude a potential role for inflammasome activation in the progression of these diseases. A role for the inflammasome in some autoimmune diseases is likely, considering the wide spectrum of endogenous danger signals that activate NLRs 18 and the role that inflammasome products, such as IL- $1 \beta$ and IL-18, can play in shaping adaptive immunity (Figure 2$)^{19}$. IL-1 $\beta$ can act on lymphocytes in several ways including upregulating IL-2 receptor expression, prolonging survival of T cells, enhancing antibody production by B cells and increasing B cell proliferation ${ }^{20,21}$. IL-1 $\beta$ and IL-18 also play a critical role in driving the differentiation and amplification of Th17 and Th1 cells, respectively ${ }^{22-24}$. So, in general, IL-1 $\beta$ and IL-18 amplify T and B cell responses and might serve as a crucial link translating NLR activation into adaptive immune responses. Here, we focus on the potential role of inflammasomes in autoimmune disorders and highlight the importance of future research in this field. An overview of current links between the inflammasome and autoimmunity will facilitate the investigation of its importance for clinical care, potentially identifying new therapeutic targets for several autoimmune disorders.

\section{Rheumatoid arthritis}

RA is a self-perpetuating autoimmune disease that can affect several tissues and organs but principally leads to chronic inflammation of the synovial membrane of joints causing pain and swelling and which may result in irreversible joint destruction. Although the precipitating causes of RA are unknown, autoimmunity plays a critical role in progression of the disease, as both $\mathrm{T}$ cells and autoantibodies are involved ${ }^{25}$. Clinical trials exploring the possibility of treating RA by blocking IL-1 activity with anakinra found reduced joint swelling, pain and inflammatory blood markers strategy ${ }^{26}$, but the use of anakinra is less favored because other anticytokine therapies are more effective ${ }^{27}$. Nevertheless, IL-1 blockade was moderately effective, and inhibition of the inflammasome could prove to be a more effective therapeutic target than specific IL-1 blockade as inflammasome inhibition would block IL-1 and IL-18 activity, both of which play a role in disease progression ${ }^{27,} 28$.

The evidence linking the inflammasome and RA pathogenesis is somewhat ambiguous; an epistatic relationship was reported whereby dual polymorphisms in the NLRP3 and CARD8 genes caused increased IL- $1 \beta$ production and was associated with an increased susceptibility and severity of disease in RA patients ${ }^{29}$, but a prospective study from the same research group did not find any association of the NLRP3 single nucleotide polymorphisms (SNPs) alone with either susceptibility to RA or the incidence or severity of early RA ${ }^{30}$. Furthermore, a difference in NLRP3 expression is not responsible for differences in the nature and extent of synovial inflammation seen in RA and osteoarthritis patients 31 . Nevertheless, these studies do not rule out the possibility of the NLRP3 inflammasome playing a functional role in RA, both in initiating and maintaining the inflammatory phases of the disorder. Patients with excessive NLRP3 activation commonly develop joint pains and arthritis 10, 32, suggesting excessive inflammasome activation could be involved in RA pathogenesis. However, the role of the NLRP3 inflammasome in the etiopathogenesis of RA requires further study.

In mice, both antigen-induced arthritis (AIA) and collagen-induced arthritis (CIA) are used to explore disease mechanisms of RA, as these models share some clinical and pathological features with RA in humans. However, these animal models do differ from RA in several aspects, and therapies effective in these conditions might not be as useful in a clinical 
setting. However, disease progression was reportedly independent of NLRP3 and caspase-1 in these mouse models of $\mathrm{RA}^{33}$, 34, and surprisingly, both studies identified an inflammasome-independent role of ASC. The mechanisms reported by which ASC contributed to the development of arthritis were different in these two studies. ASC was required for cytokine production and $\mathrm{T}$ cell proliferation in one report34, whereas a dendritic cell-intrinsic role for ASC in lymphocyte activation was reported in the other study33. Therefore, in mouse models of arthritis, the NLRP3 inflammasome does not play a role in disease progression. However, these are imperfect models of RA disease mechanisms in humans, and further clinical research is required to fully understand the role of inflammasomes in RA.

\section{Multiple sclerosis}

The initiation and progression of multiple sclerosis (MS) is driven by autoreactive T cells targeting oligodendrocytes. MS is a heterogeneous condition both in clinical presentation and disease duration. Although both Th1 and Th17 cells drive the pathogenesis of $\mathrm{MS}^{35}$, innate immune cells also play a significant role in disease progression36. Cytokines activated by the inflammasome, IL-1 $\beta$ and IL-18, play a major role in the differentiation and survival of Th17 and Th1 cells, respectively19, 22, 37-40, suggesting a possible role for inflammasome activation in the progression of MS.

To date, there has not been an association between inflammasome-forming NLR mutations or polymorphisms and MS, but this does not rule out a possible role for the inflammasome(s) in MS progression. Additionally, a recent case report described lesions in the brain of a patient suffering from MWS that resembled those seen in MS patients ${ }^{41}$. Although, this is not a direct link with MS, it does suggest the overactivation of the NLRP3 inflammasome can cause CNS inflammation, which could be involved in MS progression.

In experimental autoimmune encephalomyelitis (EAE), a mouse model of MS, caspase-1 activation was required for development of EAE through the production of inflammatory cytokines Il-1 $\beta$ and IL- $18^{42}$. However, the data on the role of the NLRP3 inflammasome in EAE progression are conflicting; one report found a role for the NLRP3 inflammasome in development of Th1 and Th17 cells and EAE progression43, whereaas another report did not find a role for the NLRP3 inflammasome in EAE progression, but did find an inflammasome-independent role for ASC44. Lymphocyte survival was significantly impaired in the absence of ASC, and ASC-deficient mice were resistant to EAE. The model and methodology were the same for the two reports; however, the gut microbiota can greatly influence adaptive immunity, and especially Th17 responses, in mouse models of autoimmunity45. Therefore, it is possible that there are differences in the mechanisms of disease pathogenesis in these two independent mouse colonies in different locations. Future studies in other laboratories examining EAE progression in homozygous knockout $N L R P 3^{-1-}$ mice could clarify this discrepancy. However, there is substantial evidence in animal models for inflammasome involvement, in that caspase- 1 activation plays a critical role in EAE pathogenesis. Clinically, caspase-1 expression is elevated in MS plaques and in the peripheral blood mononuclear cells (PBMCs) of MS patients compared to healthy brain sections and PBMCs, respectively ${ }^{46,}$ 47. Future studies exploring the efficacy of a caspase-1 inhibitor in primate models of MS would be useful in elucidating a causal role for inflammasome activation in MS. Nevertheless, the role of inflammasome activation and any NLR involvement in MS progression remains in question.

\section{Type 1 diabetes}

The progressive destruction of insulin-secreting islet cells of the pancreas by autoreactive $\mathrm{T}$ cells and autoantibodies leads to type 1 diabetes (T1D) $)^{48}$, 49. Insulin replacement therapy is 
used to manage T1D, but the disease and related complications, including cardiovascular disease, diabetic retinopathy and diabetic neuropathy, remain a serious burden on human health 50, 51. The mechanisms causing T1D and its complications are not fully understood. However, based on results showing that IL- $1 \beta$ can cause pancreatic beta cell apoptosis ${ }^{52}$, a multicenter, randomized clinical trial was recently launched that will investigate the effect of IL-1 antagonism on beta cell function in recent onset T1D patients ${ }^{53}$

(www.clinicaltrials.gov). Despite extensive work investigating the role of IL-1 in T1D, it remains unknown whether NLRP3 inflammasome activation contributes to pathogenesis.

A recent study analyzing 1,084 patients with T1D and 3,273 healthy patients found a strong correlation with a SNP in NLRP1, rs 12150220 , and T1D ${ }^{54}$. However, the association did not reach genome-wide significance. This SNP is located in the NACHT region of NLRP1, but the effect of this SNP on NLRP1 inflammasome function is unknown. A more recent study did not find any association between this NLRPI SNP and T1D in a pediatric population in Brazil, but this study was only comprised of 196 patients with T1D and 192 healthy individuals ${ }^{55}$. However, a SNP in NLRP3, rs 10754558, was associated specifically with T1D susceptibility in this pediatric population in Brazil ${ }^{55}$. This particular NLRP3 SNP was recently associated with mRNA stability ${ }^{56}$ and, therefore, it is possible that stable expression of NLRP3 could be protective against T1D. However, the effect of this SNP on inflammasome activation or the consequent secretion of IL- $1 \beta$ and IL-18 are, as yet, unknown, and the reason that the $N L R P 3$ rs $10754558 \mathrm{G}$ allele was less frequent in patients with T1D is also unknown.

The role of the NLRP1 or NLRP3 inflammasomes in nonobese diabetic (NOD) mice, a strain that develops T1D spontaneously, has not been studied. Future studies exploring the effect of NLRPI and NLRP3 gene deletion or mutations in NOD mice could be valuable. However, caspase-1 is not required for development of T1D in the NOD mouse ${ }^{57}$, suggesting that deficiency of either NLRP1 or NLRP3 would not alter the development of T1D in NOD mice, and moreover, disease mechanisms in the NOD model do not always correlate with T1D pathogenesis. Future studies inserting the rs12150220 NLRP1 or rs 10754558 NLRP3 SNP in NOD transgenic mice might provide a mechanistic explanation for its association with T1D. Furthermore, although caspase-1 is not involved in the development of T1D in NOD mice, it is involved in the degeneration of retinal capillaries in diabetes ${ }^{58}$. Whether this is through the NLRP1 or NLRP3 inflammasome is unknown, but if either inflammasome is involved, then specific NLRP1 or NLRP3 inhibitors might provide a valuable therapeutic approach to prevention of diabetic retinopathy in T1D patients. A significant amount of research remains to be done in this field, but initial evidence suggests that the NLRP1 or NLRP3 inflammasome could play a role in the predisposition to development of T1D or progression of diabetic retinopathy. Therefore, future work may provide novel therapeutic strategies for T1D or its complications.

\section{Systemic lupus erythematosus}

Systemic lupus erythematosus (SLE) is an autoimmune disease driven by an immune response against ubiquitous self-antigens. Autoantibodies against nuclear antigens, especially double-stranded DNA are commonly detected. To date, there has not been any association with inflammasome-forming NLR mutations or polymorphisms and lupus. However, leukocytes from SLE patients have increased AIM2 expression, but this study did not find a correlation between AIM2 expression and SLE Disease Activity 2000 scores $^{59}$. A preliminary study showed that IL-1 blockade with anakinra provided therapeutic benefit for patients with SLE60. Additionally, both IL-1 $\beta$ and IL-18 are involved in disease progression in the mouse model of lupus, suggesting the involvement of at least one inflammasome61, 62. Chloroquine, used widely for SLE, decreases aberrant NLRP3 expression during disease 
progression, a mechanism possibly related to its therapeutic effect ${ }^{63}$. If inflammasome activation plays a role in lupus progression, then AIM2 is a good candidate to be the main NLR involved, based on its ability to recognize cytoplasmic DNA ${ }^{64}$. However, the involvement of AIM2 or any other inflammasome-forming NLRs has not been studied at this point and it is possible for multiple inflammasomes to be involved in the activation of IL-1 $\beta$ and IL-18. Future studies deleting the NLRP3, NLRP1 or AIM2 genes in transgenic lupus-prone mice and examining disease progression will help elucidate the role of the inflammasome in lupus, and could provide initial evidence for a new therapeutic strategy for its management

\section{Celiac disease}

Celiac disease (CD) is an inherited disease characterized by destruction of the lining of the small intestine that is triggered by eating gluten, gliadin and other proteins found in wheat, barley and rye ${ }^{65}$. Several characteristics are shared between $C D$ and inflammatory bowel disease (IBD), but CD is diagnosed by a defined dietary trigger, the presence of HLA-DQ2 or HLA-DQ8 genotype and the presence of autoantibodies to tissue transglutaminase. Several disorders might be associated with CD susceptibility, including several different autoimmune diseases, such as RA and SLE. Indeed, a case report describes a child who was diagnosed with both a cryopyrinopathy and CD66. Although this occurrence could be coincidental, it is also possible that excessive IL-1 $\beta$ and IL-18 production due to a mutation in NLRP 3 could have contributed to the generation of gliadin-reactive T cells. Furthermore, in a pediatric population of CD patients, the NLRP3 SNP (rs10754558 G allele) was less frequently observed in $\mathrm{CD}$ patients compared to healthy patients55; interestingly, this same result was also observed in patients with T1D55. As the functional consequences of this SNP are unknown, it is as yet too early to know if NLRP3 is involved in predisposition to or progression of CD. Nevertheless, downstream products of NLRP3 inflammasome activation, such as IL-1 $\beta$, control the IL-23 response to gliadin, and therefore affect Th17 cell differentiation and survival67. Additionally, the receptor for the IL-18 protein (IL18RAP and IL18R1) is a susceptibility locus for CD, and IL-18 is believed to play a role in the pathogenesis of disease by inducing Th1 responses68, 69. Therefore, it seems possible that the NLRP3 inflammasome could be involved in CD pathogenesis; studies of NLRP3 knockouts or insertion of the NLRP3 rs $10754558 \mathrm{G}$ allele SNP onto HLA-DQ8 transgenic mice, a mouse model of CD, would help clarify the role of the NLRP3 inflammasome in its pathogenesis.

\section{Vitiligo and vitiligo-associated multiple autoimmune disease}

Vitiligo is an autoimmune disease caused by the T cell-mediated destruction of melanocytes, presenting as white patches on the skin due to a loss of pigment. The mechanisms underlying disease progression are unclear, but a wide array of stress factors can provoke an autoimmune response to melanocytes. However, only $0.5-1 \%$ of individuals will develop progressive depigmentation in response to stress factors, implicating a hereditary predisposition in a subset of the population ${ }^{70}$. Additionally, as with several other autoimmune disorders, patients with vitiligo often are afflicted with concurrent autoimmune disorders. A recent association study found a strong association of $N L R P 1$ variants with vitiligo alone and vitiligo-associated multiple autoimmune diseases ${ }^{71}$. The functional effects of the NLRP1 SNP variants are unknown, and therefore the mechanism(s) linking NLRP1 variations and vitiligo remain(s) unknown. However, NLRP1 is highly expressed in both T cells and Langerhans' cells, a distribution pattern that suggests a potential role for NLRP1 in skin autoimmunity ${ }^{72}$. Studies examining the effects of $N L R P 1, N L R P 3$ or caspase-1 gene deletion in a recently described mouse model of autoimmune vitiligo ${ }^{73}$, would help determine the potential role of the inflammasomes in vitiligo. 


\section{Autoimmune Addison's disease}

Addison's disease (AAD) is an autoimmune condition associated with immune destruction of the adrenal cortex resulting in a deficiency of mineralocorticoid, glucocorticoid and adrenal androgen production. The pathogenesis of autoimmune AAD, which is the most common disease of the adrenal cortex, is a complex process, influenced by both genetic and environmental factors. Based on the association of NLRPI SNPs with other autoimmune disorders, a study examined the correlation of six NLRP1 SNPs with AAD (101 AAD and 254 healthy individuals), and the frequency of the rs12150220 SNP in NLRPI was significantly increased among AAD patients ${ }^{74}$. However, based on the low prevalence of $\mathrm{AAD}$, the study was unable to examine a sufficiently large cohort of patients to reach genome-wide significance. This study demonstrates an association of the NLRPI SNPs and genetic susceptibility to AAD, but the exact role of the NLRP1 inflammasome in AAD progression remains unclear.

\section{Concluding Remarks}

Our understanding of NLRs and their role in human diseases has expanded exponentially in recent years. NLRP3 inflammasome activation is critically involved in autoinflammatory disorders, and a role for this particular inflammasome has been shown in animal models of several other diseases with an inflammatory component (Table 1). However, despite the potential of the NLRP3 inflammasome to affect adaptive immune responses via IL-1 $\beta$ and IL-18 production, its role in autoimmune disorders has not been studied extensively. Several reports have linked various polymorphisms in different components of inflammasomeforming NLRs, including NLRP3 and NLRP1, to autoimmunity. Future work elucidating the effects of these identified SNPs on gene transcription, mRNA stability or protein function will provide substantial progress in determining the role of the inflammasomes in disease progression. Additionally, activators of NLRs continue to be determined, and the discovery of the range of molecules capable of activating the inflammasomes could provide breakthroughs in our understanding of specific disease progression. For example, the finding that gout-associated urate crystals are involved in activating the NLRP3 inflammasome was a significant advance in understanding the pathogenesis of gout and its treatment ${ }^{75}$. Our understanding of NLRs and the inflammasomes in autoimmunity is still rather elementary, but preliminary studies suggest a potential role of inflammasome activation in at least some autoimmune disorders. Further progress in this research field could open up new strategies in treating autoimmune diseases and their complications.

\section{Acknowledgments}

We acknowledge researchers who have contributed to this field whose work was not cited or was cited through review articles because of space limitations. This work is supported by National Institute of Health Grants AR056296 and AI088177, a NIAMS Centers of Excellence for Influenza Research and Surveillance (CEIRS) grant and the American Lebanese Syrian Associated Charities (ALSAC). M. F. McDermott is supported by an FP7HEALTH-2007-2.4.4-1 grant and an Arthritis Research UK project grant.

\section{References}

1. Kanneganti TD, et al. Intracellular NOD-like receptors in host defense and disease. Immunity 2007;27:549-559. [PubMed: 17967410]

2. Ting JP, et al. The NLR gene family: a standard nomenclature. Immunity 2008;28:285-287. [PubMed: 18341998]

3. Bauer C, et al. Colitis induced in mice with dextran sulfate sodium (DSS) is mediated by the NLRP3 inflammasome. Gut 2010;59:1192-1199. [PubMed: 20442201] 
4. Duewell P, et al. NLRP3 inflammasomes are required for atherogenesis and activated by cholesterol crystals. Nature 2010;464:1357-1361. [PubMed: 20428172]

5. Zaki MH, et al. The NLRP3 inflammasome protects against loss of epithelial integrity and mortality during experimental colitis. Immunity 2010;32:379-391. [PubMed: 20303296]

6. Zhou R, et al. Thioredoxin-interacting protein links oxidative stress to inflammasome activation. Nat Immunol 2010;11:136-140. [PubMed: 20023662]

7. McGonagle D, et al. An integrated classification of pediatric inflammatory diseases, based on the concepts of autoinflammation and the immunological disease continuum. Pediatr Res 2009;65:38R45R.

8. Dickie LJ, et al. Periodic fever syndrome and autoinflammatory diseases. F1000 Med Rep 2010;2

9. Shinkai K, et al. Cryopyrin-associated periodic syndromes and autoinflammation. Clin Exp Dermatol 2008;33:1-9. [PubMed: 17927785]

10. Aksentijevich I, et al. The clinical continuum of cryopyrinopathies: novel CIAS1 mutations in North American patients and a new cryopyrin model. Arthritis Rheum 2007;56:1273-1285. [PubMed: 17393462]

11. Hoffman HM, et al. Prevention of cold-associated acute inflammation in familial cold autoinflammatory syndrome by interleukin-1 receptor antagonist. Lancet 2004;364:1779-1785. [PubMed: 15541451]

12. Neven B, et al. Cryopyrinopathies: update on pathogenesis and treatment. Nat Clin Pract Rheumatol 2008;4:481-489. [PubMed: 18665151]

13. Joosten LA, et al. Fatty acids engagement with TLR2 drive IL-1beta production via ASCcaspase- 1 activation by urate crystals in gouty arthritis. Arthritis Rheum. 2010

14. Martinon F, Glimcher LH. Gout: new insights into an old disease. J Clin Invest 2006;116:20732075. [PubMed: 16886051]

15. So A, et al. A pilot study of IL-1 inhibition by anakinra in acute gout. Arthritis Res Ther 2007;9:R28. [PubMed: 17352828]

16. Olson JK, Miller SD. The innate immune response affects the development of the autoimmune response in Theiler's virus-induced demyelinating disease. J Immunol 2009;182:5712-5722. [PubMed: 19380818]

17. Firestein GS, Zvaifler NJ. How important are T cells in chronic rheumatoid synovitis?: II. T cellindependent mechanisms from beginning to end. Arthritis Rheum 2002;46:298-308. [PubMed: 11840432]

18. Schroder K, Tschopp J. The inflammasomes. Cell 2010;140:821-832. [PubMed: 20303873]

19. Sims JE, Smith DE. The IL-1 family: regulators of immunity. Nat Rev Immunol 2010;10:89-102. [PubMed: 20081871]

20. Ben-Sasson SZ, et al. IL-1 acts directly on CD4 T cells to enhance their antigen-driven expansion and differentiation. Proc Natl Acad Sci U S A 2009;106:7119-7124. [PubMed: 19359475]

21. Maliszewski CR, et al. Cytokine receptors and B cell functions. I. Recombinant soluble receptors specifically inhibit IL-1- and IL-4-induced B cell activities in vitro. J Immunol 1990;144:30283033. [PubMed: 2139075]

22. Chung Y, et al. Critical regulation of early Th17 cell differentiation by interleukin-1 signaling. Immunity 2009;30:576-587. [PubMed: 19362022]

23. Guo L, et al. IL-1 family members and STAT activators induce cytokine production by Th2, Th17, and Th1 cells. Proc Natl Acad Sci U S A 2009;106:13463-13468. [PubMed: 19666510]

24. Hata H, et al. IL-18 together with anti-CD3 antibody induces human Th1 cells to produce Th1- and Th2-cytokines and IL-8. Int Immunol 2004;16:1733-1739. [PubMed: 15477227]

25. Manzo A, et al. Secondary and ectopic lymphoid tissue responses in rheumatoid arthritis: from inflammation to autoimmunity and tissue damage/remodeling. Immunol Rev 2010;233:267-285. [PubMed: 20193005]

26. Dinarello CA. Blocking IL-1 in systemic inflammation. J Exp Med 2005;201:1355-1359. [PubMed: 15867089]

27. Goldbach-Mansky R. Blocking interleukin-1 in rheumatic diseases. Ann N Y Acad Sci 2009;1182:111-123. [PubMed: 20074280] 
28. Lotito AP, et al. Interleukin-18 in chronic joint diseases. Autoimmun Rev 2007;6:253-256. [PubMed: 17317618]

29. Kastbom A, et al. Genetic variation in proteins of the cryopyrin inflammasome influences susceptibility and severity of rheumatoid arthritis (the Swedish TIRA project). Rheumatology (Oxford) 2008;47:415-417. [PubMed: 18263599]

30. Kastbom A, et al. CARD8 p.C10X polymorphism is associated with inflammatory activity in early rheumatoid arthritis. Ann Rheum Dis 2010;69:723-726. [PubMed: 19443463]

31. Kolly L, et al. Expression and function of the NALP3 inflammasome in rheumatoid synovium. Immunology. 2009

32. Liu-Bryan R. Intracellular innate immunity in gouty arthritis: role of NALP3 inflammasome. Immunol Cell Biol 2010;88:20-23. [PubMed: 19935768]

33. Ippagunta SK, et al. Inflammasome-independent role of apoptosis-associated speck-like protein containing a CARD (ASC) in T cell priming is critical for collagen-induced arthritis. J Biol Chem 2010;285:12454-12462. [PubMed: 20177071]

34. Kolly L, et al. Inflammatory role of ASC in antigen-induced arthritis is independent of caspase-1, NALP-3, and IPAF. J Immunol 2009;183:4003-4012. [PubMed: 19717512]

35. Goverman J. Autoimmune T cell responses in the central nervous system. Nat Rev Immunol 2009;9:393-407. [PubMed: 19444307]

36. Gandhi R, et al. Role of the innate immune system in the pathogenesis of multiple sclerosis. J Neuroimmunol 2010;221:7-14. [PubMed: 19931190]

37. Acosta-Rodriguez EV, et al. Interleukins 1 beta and 6 but not transforming growth factor-beta are essential for the differentiation of interleukin 17-producing human T helper cells. Nat Immunol 2007;8:942-949. [PubMed: 17676045]

38. Kryczek I, et al. Cutting edge: opposite effects of IL-1 and IL-2 on the regulation of IL-17+ T cell pool IL-1 subverts IL-2-mediated suppression. J Immunol 2007;179:1423-1426. [PubMed: 17641006]

39. Nakanishi K, et al. Interleukin-18 regulates both Th1 and Th2 responses. Annu Rev Immunol 2001;19:423-474. [PubMed: 11244043]

40. Sutton C, et al. A crucial role for interleukin (IL)-1 in the induction of IL-17-producing T cells that mediate autoimmune encephalomyelitis. J Exp Med 2006;203:1685-1691. [PubMed: 16818675]

41. Compeyrot-Lacassagne $\mathrm{S}$, et al. Brain multiple sclerosis-like lesions in a patient with MuckleWells syndrome. Rheumatology (Oxford) 2009;48:1618-1619. [PubMed: 19828598]

42. Furlan R, et al. Caspase-1 regulates the inflammatory process leading to autoimmune demyelination. J Immunol 1999;163:2403-2409. [PubMed: 10452974]

43. Gris D, et al. NLRP3 plays a critical role in the development of experimental autoimmune encephalomyelitis by mediating Th1 and Th17 responses. J Immunol 2010;185:974-981. [PubMed: 20574004]

44. Shaw PJ, et al. Cutting Edge: critical role for PYCARD/ASC in the development of experimental autoimmune encephalomyelitis. J Immunol 2010;184:4610-4614. [PubMed: 20368281]

45. Lee YK, et al. Microbes and Health Sackler Colloquium: Proinflammatory T-cell responses to gut microbiota promote experimental autoimmune encephalomyelitis. Proc Natl Acad Sci U S A. 2010

46. Ming X, et al. Caspase-1 expression in multiple sclerosis plaques and cultured glial cells. J Neurol Sci 2002;197:9-18. [PubMed: 11997061]

47. Huang WX, et al. Increased expression of caspase-1 and interleukin-18 in peripheral blood mononuclear cells in patients with multiple sclerosis. Mult Scler 2004;10:482-487. [PubMed: 15471361]

48. Yoshida K, Kikutani H. Genetic and immunological basis of autoimmune diabetes in the NOD mouse. Rev Immunogenet 2000;2:140-146. [PubMed: 11324686]

49. Ziegler AG, Nepom GT. Prediction and pathogenesis in type 1 diabetes. Immunity 2010;32:468478. [PubMed: 20412757]

50. Deshpande AD, et al. Epidemiology of diabetes and diabetes-related complications. Phys Ther 2008;88:1254-1264. [PubMed: 18801858] 
51. Grauslund J. Long-term mortality and retinopathy in type 1 diabetes. Acta Ophthalmol 2010;88(Thesis 1):1-14. [PubMed: 20500731]

52. Atkinson MA, Eisenbarth GS. Type 1 diabetes: new perspectives on disease pathogenesis and treatment. Lancet 2001;358:221-229. [PubMed: 11476858]

53. Pickersgill LM, Mandrup-Poulsen TR. The anti-interleukin-1 in type 1 diabetes action trial-background and rationale. Diabetes Metab Res Rev 2009;25:321-324. [PubMed: 19405081]

54. Magitta NF, et al. A coding polymorphism in NALP1 confers risk for autoimmune Addison's disease and type 1 diabetes. Genes Immun 2009;10:120-124. [PubMed: 18946481]

55. Pontillo A, et al. Two SNPs in NLRP3 gene are involved in the predisposition to type-1 diabetes and celiac disease in a pediatric population from northeast Brazil. Autoimmunity. 2010

56. Hitomi Y, et al. Associations of functional NLRP3 polymorphisms with susceptibility to foodinduced anaphylaxis and aspirin-induced asthma. J Allergy Clin Immunol 2009;124:779-785. e776. [PubMed: 19767079]

57. Schott WH, et al. Caspase-1 is not required for type 1 diabetes in the NOD mouse. Diabetes 2004;53:99-104. [PubMed: 14693703]

58. Vincent JA, Mohr S. Inhibition of caspase-1/interleukin-1 beta signaling prevents degeneration of retinal capillaries in diabetes and galactosemia. Diabetes 2007;56:224-230. [PubMed: 17192486]

59. Kimkong I, et al. Expression profile of HIN200 in leukocytes and renal biopsy of SLE patients by real-time RT-PCR. Lupus 2009;18:1066-1072. [PubMed: 19762380]

60. Ostendorf B, et al. Preliminary results of safety and efficacy of the interleukin 1 receptor antagonist anakinra in patients with severe lupus arthritis. Ann Rheum Dis 2005;64:630-633. [PubMed: 15345502]

61. Calvani N, et al. Th1 cytokines in the pathogenesis of lupus nephritis: the role of IL-18. Autoimmun Rev 2005;4:542-548. [PubMed: 16214093]

62. Voronov E, et al. IL-1 beta-deficient mice are resistant to induction of experimental SLE. Eur Cytokine Netw 2006;17:109-116. [PubMed: 16840029]

63. Glinsky GV. SNP-guided microRNA maps (MirMaps) of 16 common human disorders identify a clinically accessible therapy reversing transcriptional aberrations of nuclear import and inflammasome pathways. Cell Cycle 2008;7:3564-3576. [PubMed: 19001869]

64. Choubey D, et al. Interferon-Inducible p200-Family Proteins as Novel Sensors of Cytoplasmic DNA: Role in Inflammation and Autoimmunity. J Interferon Cytokine Res. 2010

65. Schuppan D, et al. Celiac disease: from pathogenesis to novel therapies. Gastroenterology 2009;137:1912-1933. [PubMed: 19766641]

66. Shaker M, et al. Association between celiac sprue and cryopyrin associated autoinflammatory disorders: a case report. Pediatr Rheumatol Online J 2007;5:12. [PubMed: 17550587]

67. Harris KM, et al. Cutting edge: IL-1 controls the IL-23 response induced by gliadin, the etiologic agent in celiac disease. J Immunol 2008;181:4457-4460. [PubMed: 18802048]

68. Garrote JA, et al. Celiac disease pathogenesis: the proinflammatory cytokine network. J Pediatr Gastroenterol Nutr 2008;47 Suppl 1:S27-S32. [PubMed: 18667914]

69. Hunt KA, et al. Newly identified genetic risk variants for celiac disease related to the immune response. Nat Genet 2008;40:395-402. [PubMed: 18311140]

70. Spritz RA. The genetics of generalized vitiligo and associated autoimmune diseases. Pigment Cell Res 2007;20:271-278. [PubMed: 17630960]

71. Jin Y, et al. NALP1 in vitiligo-associated multiple autoimmune disease. N Engl J Med 2007;356:1216-1225. [PubMed: 17377159]

72. Kummer JA, et al. Inflammasome components NALP 1 and 3 show distinct but separate expression profiles in human tissues suggesting a site-specific role in the inflammatory response. $\mathrm{J}$ Histochem Cytochem 2007;55:443-452. [PubMed: 17164409]

73. Denman CJ, et al. HSP70i accelerates depigmentation in a mouse model of autoimmune vitiligo. J Invest Dermatol 2008;128:2041-2048. [PubMed: 18337834]

74. Zurawek M, et al. A coding variant in NLRP1 is associated with autoimmune Addison's disease. Hum Immunol 2010;71:530-534. [PubMed: 20152874] 
75. Martinon F, et al. Gout-associated uric acid crystals activate the NALP3 inflammasome. Nature 2006;440:237-241. [PubMed: 16407889]

76. Villani AC, et al. Common variants in the NLRP3 region contribute to Crohn's disease susceptibility. Nat Genet 2009;41:71-76. [PubMed: 19098911]

77. Larsen CM, et al. Interleukin-1-receptor antagonist in type 2 diabetes mellitus. N Engl J Med 2007;356:1517-1526. [PubMed: 17429083]

78. Omi T, et al. An intronic variable number of tandem repeat polymorphisms of the cold-induced autoinflammatory syndrome 1 (CIAS1) gene modifies gene expression and is associated with essential hypertension. Eur J Hum Genet 2006;14:1295-1305. [PubMed: 16868559]

79. Halle A, et al. The NALP3 inflammasome is involved in the innate immune response to amyloidbeta. Nat Immunol 2008;9:857-865. [PubMed: 18604209]

80. Sutterwala FS, et al. Critical role for NALP3/CIAS1/Cryopyrin in innate and adaptive immunity through its regulation of caspase-1. Immunity 2006;24:317-327. [PubMed: 16546100]

81. Meng G, et al. A mutation in the Nlrp3 gene causing inflammasome hyperactivation potentiates Th17 cell-dominant immune responses. Immunity 2009;30:860-874. [PubMed: 19501001]

82. Brydges SD, et al. Inflammasome-mediated disease animal models reveal roles for innate but not adaptive immunity. Immunity 2009;30:875-887. [PubMed: 19501000]

83. Lee WW, et al. Regulating human Th17 cells via differential expression of IL-1 receptor. Blood 2010;115:530-540. [PubMed: 19965648] 


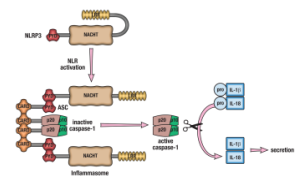

Figure 1. Activation of the inflammasome

The various inflammasomes are activated by a wide spectrum of DAMPs and PAMPs.

NLRP3 activation and inflammasome formation is depicted as an example. Once activated, the inflammasome-forming NLR undergoes a conformational change, allowing for NLR binding to ASC via the pyrin domains (PYD). ASC acts an adaptor protein, and binds to pro-caspase-1 through caspase recruitment domains (CARD). This large complex is termed the inflammasome and provides the platform for the autoactivation of caspase- 1 by proteolytic cleavage. Active caspase-1 (p20/p10) then cleaves pro-forms of IL-1 $\beta$ and IL-18, allowing for their secretion and biological activity. 


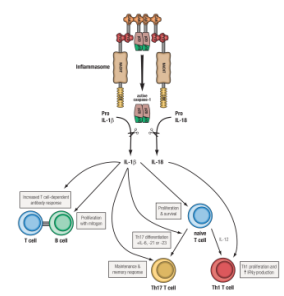

Figure 2. The influence of inflammasome activation on adaptive immunity

Activation of the inflammasome results in the cleavage of proIL-1 $\beta$ and proIL-18 into their biologically active forms, IL-1 $\beta$ and IL-18, which can shape adaptive immune responses in a number of ways. IL-18, formerly known as IFN $\gamma$-inducing factor, enhances Th1 cell proliferation and production of IFN $\gamma$; IL-1 $\beta$ can enhance naïve T cell survival and proliferation, via upregulation of the IL-2 receptor. Additionally, a role for IL-1R signaling in Th17 cell differentiation and homeostasis has recently been reported ${ }^{83}$. Humoral responses can also be enhanced by IL-1 $\beta$, either directly via increased B cell proliferation or indirectly by upregulation of co-stimulatory molecules on T cells. Therefore, activation of the inflammasome can significantly affect an adaptive immune response through the cleavage of IL-18 and IL-1 $\beta$. 
Table 1

The role of the inflammasomes in human disease

\begin{tabular}{|c|c|c|c|c|}
\hline Human disease & Cause of disease & $\begin{array}{c}\text { Link to } \\
\text { genetic } \\
\text { mutations }\end{array}$ & $\begin{array}{c}\text { Response } \\
\text { to IL-1 } \\
\text { blockade? }\end{array}$ & $\begin{array}{c}\text { Inflammasome } \\
\text { involved in } \\
\text { animal model? }\end{array}$ \\
\hline \multicolumn{5}{|l|}{$\begin{array}{c}\text { Diseases with an inflammatory } \\
\text { component }\end{array}$} \\
\hline Inflammatory Bowel Disease & Polygenic inflammation of GI tract & Yes 76 & No & $\mathrm{Yes}^{3,5}$ \\
\hline Type 2 diabetes & Polygenic/metabolic stress and inflammation & No & Yes 77 & Yes $^{6}$ \\
\hline Essential hypertension & High blood pressure without an identifiable cause & Yes $^{78}$ & Not tested & Not tested \\
\hline Alzheimer's disease & Degenerative brain disorder & No & Not tested & Yes 79 \\
\hline Atherosclerosis & $\begin{array}{l}\text { Artery wall thickening as a result of build-up of fatty } \\
\text { materials }\end{array}$ & No & Not tested & $\mathrm{Yes}^{4}$ \\
\hline Contact allergic dermatitis & Sensitizing allergens & Yes 10 & Not tested & Yes 80 \\
\hline \multicolumn{5}{|l|}{ Autoinflammatory diseases } \\
\hline MWS & NLRP3 mutation & Yes 10 & Yes $^{12}$ & Yes $^{81}$ \\
\hline FCAS & NLRP3 mutation & Yes $^{10}$ & Yes $^{12}$ & Yes $^{82}$ \\
\hline CINCA & NLRP3 mutation & Yes $^{10}$ & Yes $^{12}$ & Not tested \\
\hline \multicolumn{5}{|l|}{ Autoimmune Diseases } \\
\hline Type 1 diabetes & Self destruction of pancreatic $\beta$ cells & $\mathrm{Yes}^{55}$ & Not tested & Not tested \\
\hline Multiple sclerosis & Self destruction of oligodendrocytes & No & In progress & $\mathrm{Yes}^{43} / \mathrm{No}^{44}$ \\
\hline Systemic lupus erythematosus & $\begin{array}{c}\text { Targeting of ubiquitous, mostly intra-nuclear self- } \\
\text { antigens }\end{array}$ & No & Yes $^{60}$ & Not tested \\
\hline Vitiligo & Self destrucion of melanocytes & Yes $^{71}$ & Not tested & Not tested \\
\hline Addison's disease & $\begin{array}{c}\text { Destruction of the adrenal cortex resulting in impaired } \\
\text { cortisol production }\end{array}$ & $\mathrm{Yes}^{54}$ & Not tested & Not tested \\
\hline Celiac disease & $\begin{array}{c}\text { Destruction of the small intestine lining triggered by } \\
\text { eating gluten }\end{array}$ & $\mathrm{Yes}^{55}$ & Not tested & Not tested \\
\hline Rheumatoid arthritis & Self destruction of the synovial joints & Yes $^{29}$ & Yes $^{27}$ & $\mathrm{No}^{33}$ \\
\hline
\end{tabular}

